\title{
Distribution of $\eta$ Precipitinogen in Mycobacteria
}

\author{
TOSHIHIRO HIRAI \\ Department of Tuberculosis Research I, Research Institute for Microbial Diseases, Osaka University, 3-1, Yamadaoka, \\ Suita City, Osaka, Japan
}

\begin{abstract}
Analysis with anti-Mycobacterium bovis BCG and anti-M. bovis Ravenel sera, absorption tests with anti$\eta$ antibody, and the failure to detect any factor that affected $\eta$ antigenic activity in $M$. bovis BCG or strain H37Ra confirmed the absence of $\eta$ precipitinogen in $M$. bovis BCG and Ravenel. No $\eta$-positive strains were found among 7 strains of $M$. bovis, 19 representative strains of 15 mycobacterial species other than Mycobacterium tuberculosis and $M$. bovis, or 13 niacin-negative strains of mycobacteria isolated from patients. However, $\eta$ activity was detected in all 9 laboratory strains of $M$. tuberculosis tested, including virulent and avirulent strains, and in 29 of 30 niacin-positive strains isolated from patients, including 15 strains with single or multiple resistance to seven drugs.
\end{abstract}

Co-workers and I reported previously that a precipitinogen, designated $\eta$, was detected in sonic extracts of Mycobacterium tuberculosis strains H37Rv and H37Ra but not in Mycobacterium bovis strains BCG and Ravenel, as determined by agar gel immunodiffusion (4). We also described reproducible procedures for preparing and detecting $\eta$ and a system for $\eta$ identification in which we used purified $\eta$ precipitinogen and its antiserum as a reference (5). $M$. tuberculosis and $M$. bovis are known to be antigenically distinct from other species of mycobacteria, but $M$. tuberculosis has generally been thought to share all antigens with strains of $M$. bovis $(1,7,9,13)$. However, $\eta$ precipitinogen, which is not detectable in $M$. bovis, may be species specific for $M$. tuberculosis.

In this study to determine whether $\eta$ precipitinogen is species specific for $M$. tuberculosis, I first examined $M$. bovis extracts for the occurrence of $\eta$ precipitinogen by using methods other than those described previously (5). I then examined the distribution of $\eta$ precipitinogen in various species of Mycobacterium, including fresh isolates and drugresistant strains.

\section{MATERIALS AND METHODS}

Bacterial strains. The laboratory strains of $M$. tuberculosis and $M$. bovis used and the various nontuberculous mycobacterial species examined are listed in Table 1. All laboratory mycobacterial strains were supplied by K. Shoji, Hyogo Medical College. Two wild strains of $M$. bovis were provided by H. Takahashi, National Institute of Health, Tokyo, Japan. Mycobacterial strains freshly isolated from patient sputa were kindly provided by Kazuo Yoshida, AntiTuberculosis Association, Osaka Branch, Osaka, Japan, after drug resistance assays were performed against streptomycin, isonicotinic acid hydrazide (INH), para-amino-salicylic acid, kanamycin, ethionamide, ethambutol, and rifampin. For comparative purposes, I selected strains from patients to include as many niacin test-negative strains and as many drug-resistant, niacin-positive strains as possible. Of 30 niacin test-positive strains from patients, 15 were singly or multiply drug resistant.

The nomenclature used for representative (standard) strains of mycobacterial species is that given in Bergey's Manual of Determinative Bacteriology, 8th ed. (8).

Bacterial culture. Organisms grown as stock cultures on Ogawa medium (5) were inoculated onto glycerol-brothpotato medium (5), and the organisms ( 1 to $2 \mathrm{~g}$, wet weight) obtained after incubation at $37^{\circ} \mathrm{C}$ for 2 to 3 weeks were collected and stored at $-20^{\circ} \mathrm{C}$.

Detection of $\boldsymbol{\eta}$ precipitinogen. The methods used for preparing cell extracts from frozen cells and detecting $\eta$ precipitinogen by immunodiffusion and immunoelectrophoresis have been described previously (5). The buffer used for $\eta$ extraction was composed of $0.1 \mathrm{M}$ phosphate buffer (deferrated, $\mathrm{pH} 7.8$ ) containing $1 \mathrm{mM}$ trisodium ethylenediamine tetraacetate, $0.5 \mathrm{mM}$ ethylenediamine-di( $o$-hydroxyphenolacetic acid), and $0.02 \mathrm{M}$ sodium acetate. The frozen cells, which were suspended in the buffer described above (volume, about $3 \mathrm{ml}$ ), were sonicated twice for $5 \mathrm{~min}$ with a 5 min cooling interval. The clear supernatant obtained by centrifuging the sonicate twice at $14,500 \times g$ for $40 \mathrm{~min}$ was filtered through a membrane filter $(0.2 \mu \mathrm{m}$; EG type; Millipore Corp., Bedford, Mass.). For use as a $\eta$ precipitinogen reference in agar gel immunodiffusion, the partially purified $\eta$ described previously (5) was purified further by column chromatography on phenyl-Sepharose (Pharmacia Fine Chemicals, Uppsala, Sweden) (data not shown).

Anti- $\boldsymbol{M}$. bovis sera. The methods used for preparing complete Freund adjuvant containing mycobacteria from which the antigens to be injected were derived and the immunization procedures used have been described previously (5). Six rabbit anti- $M$. bovis sera were prepared by immunization with five different preparations of $M$. bovis BCG extract and with an extract of $M$. bovis strain Ravenel. Another anti- $M$. bovis serum was prepared as follows. A supernatant of $M$. bovis $\mathrm{BCG}$ sonicates was obtained by centrifugation twice at $14,500 \times g$ for $60 \mathrm{~min}$. This preparation was further separated into supernatant and pellet components by ultracentrifugation at $10^{5} \times g$ for $120 \mathrm{~min}$. The resulting pellet $(5 \mathrm{mg}$ of protein) was suspended in a small portion of the supernatant ( $5 \mathrm{mg}$ of protein) to give an antigen preparation rich in particulate fraction, and this was used for immunizing rabbits.

Niacin test. The niacin reaction was performed by the method of Konno (6) with the modification of Wayne and Doubek (12), using one or two well-grown cultures on Ogawa medium.

\section{RESULTS}

Confirmation that $\boldsymbol{\eta}$ antigenic activity was not detectable in $M$. bovis BCG. Co-workers and I reported previously that no $\eta$ band was detectable in $M$. bovis BCG extracts. To test whether this observation might reflect an $\eta$ defect in $M$. 
TABLE 1. Distribution of $\eta$ precipitinogen in various laboratory strains of mycobacteria

\begin{tabular}{|c|c|c|c|}
\hline $\begin{array}{l}\text { Species or } \\
\text { subspecies }\end{array}$ & Strain & $\eta$ Precipitinogen & Niacin test ${ }^{a}$ \\
\hline \multirow[t]{9}{*}{ M. tuberculosis } & H37Rv (= TMC 102) & + & ++ \\
\hline & H37Rv (Biken) & + & ++ \\
\hline & H37Ra (Biken) & + & ++ \\
\hline & Aoyama-B & + & ++ \\
\hline & Imamura no. 1 (Kimoji) & + & ++ \\
\hline & Imamura no. 6 (Toda) & + & ++ \\
\hline & Imamura no. 6 (Toda, $\operatorname{atn}^{b}$ ) & + & ++ \\
\hline & Kurono & + & + \\
\hline & H37Rv(Biken; INH-resistant) & + & $+t$ \\
\hline \multirow[t]{5}{*}{ M. bovis } & ATCC $19210^{\mathrm{T}}$ & - & + \\
\hline & $\mathrm{BCG}(=\mathrm{ATCC} 19274)$ & - & + \\
\hline & BCG (Biken) & - & + \\
\hline & TC50 & - & - \\
\hline & Ravenel & - & ++ \\
\hline M. microti & ATCC $19422^{\mathrm{T}}$ & - & + \\
\hline M. kansasii & ATCC $12478^{\mathrm{T}}$ & - & - \\
\hline \multirow[t]{2}{*}{ M. scrofulaceum } & ATCC 19275 & - & - \\
\hline & ATCC $19981^{\mathrm{T}}$ & - & - \\
\hline M. gordonae & ATCC 19277 & - & - \\
\hline M. intracellulare & ATCC 15985 & - & - \\
\hline \multirow[t]{2}{*}{ M. avium } & ATCC 15769 & - & - \\
\hline & Kirchberg & - & - \\
\hline M. xenopi & ATCC 19276 & - & - \\
\hline M. ulcerans & ATCC $19423^{\mathrm{T}}$ & - & + \\
\hline$M$. terrae & ATCC $15755^{\mathrm{T}}$ & - & - \\
\hline M. nonchromogenicum & ATCC 19531 & - & - \\
\hline M. chelonei subsp. chelonei & NCTC 946 & - & - \\
\hline M. chelonei subsp. abscessus & ATCC $19977^{\mathrm{T}}$ & - & - \\
\hline$M$. fortuitum & ATCC $6841^{\mathrm{T}}$ & - & - \\
\hline M. phlei & ATCC 19249 & - & - \\
\hline \multirow[t]{2}{*}{ M. smegmatis } & ATCC 607 & - & - \\
\hline & ATCC 14468 & - & - \\
\hline
\end{tabular}

${ }^{a}+$, Weakly positive (pink); ++ , strongly positive (deep red).

${ }^{b}$ atn, Attenuated.

bovis $\mathrm{BCG}$, I examined the following possibilities: (i) whether $\eta$ was present in an aggregated form or a nondiffusible form after immunodiffusion in an agar gel, and (ii) whether a factor was present that affected the detection of $\eta$ precipitinogen (i.e., a factor that inactivated $\eta$ in $M$. bovis BCG or produced an active form of $\eta$ in $M$. tuberculosis $\mathrm{H} 37 \mathrm{Ra}$ ).

None of the seven antisera against extracts or sonicates of $M$. bovis BCG or Ravenel formed a $\eta$ band in the $\eta$ detection system. Figure 1 shows an example of these experiments.

As Fig. 2 shows, $M$. bovis BCG extracts and sonicates did not absorb anti- $\eta$ antibody in antiserum against partially purified $\eta$ preparation with antigen excess (Fig. $2 \mathrm{~A}$ ), whereas strain $\mathrm{H} 37 \mathrm{Ra}$ extracts absorbed this anti- $\eta$ antibody completely (Fig. 2B).

To detect the presence of a factor(s) affecting $\eta$ activity in strain H37Ra or BCG, a sonic extract prepared from a mixture of strain H37Ra and BCG cells (1:1) was tested, as were strain BCG and H37Ra cells (Fig. 3). The $\eta$ band formed with the extract prepared from the mixture was a little closer to the antigen well, as the strain $\mathrm{H} 37 \mathrm{Ra}$ cell concentration was diluted by one-half with the $M$. bovis BCG cell suspension; the $\eta$ band was in a position that corresponded to the position expected from the strain H37Ra cell content alone.

Distribution of $\boldsymbol{\eta}$ precipitinogen in laboratory strains of various species of mycobacteria. Table 1 summarizes the results obtained by using immunodiffusion and rocket line immunoelectrophoresis to detect the presence of $\eta$ precipitinogen in sonic extracts of various laboratory strains of mycobacteria (Fig. 4). The results of niacin tests on strains of $M$. tuberculosis and $M$. bovis are also shown. The relationship of $\eta$ precipitinogen to the niacin reaction of freshly isolated bacteria is described below. As expected, all strains of $M$. tuberculosis gave a positive niacin reaction. However, all of the laboratory strains of $M$. bovis except strain TC50 gave various degrees of niacin reaction.

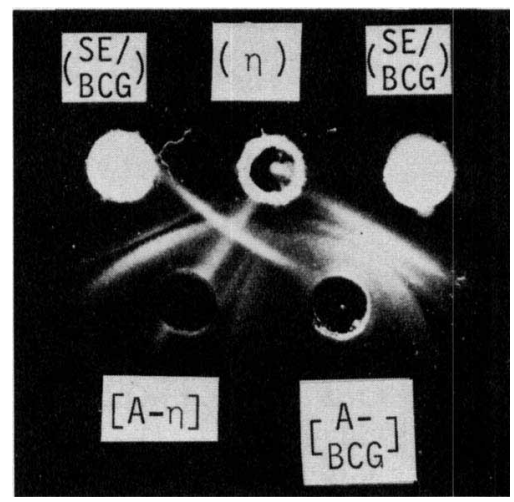

FIG. 1. Immunodiffusion pattern demonstrating the absence of anti- $\eta$ antibody in anti- $M$. bovis BCG extract serum ([A-BCG]). [A$\eta]$, Anti- $\eta$ (partially purified) serum; (SE/BCG), sonic extracts of $M$. bovis BCG cells $(5 \mathrm{mg}$ of protein per $\mathrm{ml}) ;(\eta)$, partially purified preparation of precipitinogen $\eta(20 \mu \mathrm{g}$ of protein per $\mathrm{ml})$. The antigen wells contained $10 \mu l$ of antigen solution. 


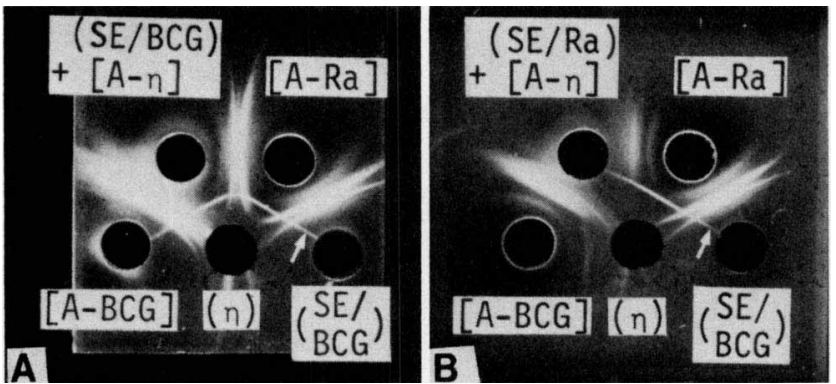

FIG. 2. Immunodiffusion patterns demonstrating that anti- $\eta$ antibody was not absorbed with $M$. bovis BCG extract (A) but was absorbed with strain H37Ra extract (B). [A-Ra], Anti-strain H37Ra extract serum; [A-BCG], anti- $M$. bovis BCG extract serum; (SE/ Ra) $+[A-\eta]$, anti- $\eta$ (partially purified) serum absorbed with sonic extract of strain $\mathrm{H} 37 \mathrm{Ra}$; (SE/BCG) $+[\mathrm{A}-\eta]$, anti- $\eta$ (partially purified) serum absorbed with sonic extract of $M$. bovis BCG; $(\eta)$, partially purified preparation of precipitinogen $\eta(20 \mu \mathrm{g}$ of protein per $\mathrm{ml})$.

All strains of $M$. tuberculosis showed $\eta$ activity, irrespective of their virulence. No mycobacteria other than $M$. tuberculosis showed $\eta$ activity.

Distribution of $\boldsymbol{\eta}$ precipitinogen in freshly isolated mycobacterial strains. I selected strains of freshly isolated mycobacteria that showed different niacin reactions and levels of drug resistance for tests of $\eta$ activity and for comparison with laboratory strains which had been passaged through many subcultures.

As Table 2 shows, all 13 freshly isolated niacin-negative strains from patients and 2 strains of $M$. bovis were $\eta$ negative. A total of 30 freshly isolated niacin-positive strains from patients, including singly and multiply drug-resistant strains, were $\eta$ positive in duplicate tests on cells from first and second cultures on potato medium. Figure 4 shows an example of the rocket line immunoelectrophoretic pattern obtained with sonic extracts of representative mycobacterial strains with partially purified $\eta$ as a reference. (This method is useful for identification of $\eta$ in many samples at once.)

\section{DISCUSSION}

Previously, co-workers and I found a precipitinogen band by using agar immunodiffusion and a $M$. tuberculosis $\mathrm{H} 37 \mathrm{Ra}$

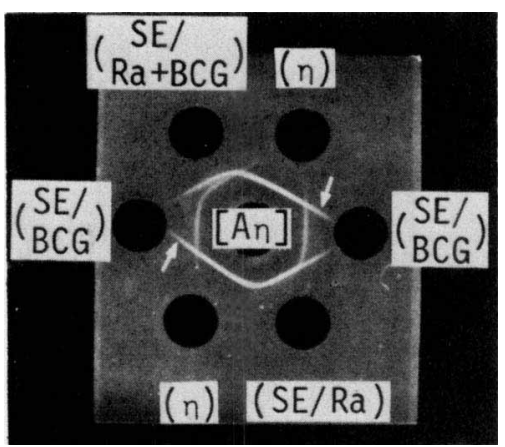

FIG. 3. Immunodiffusion pattern demonstrating that $\eta$ precipitation activity in an extract was not affected by the presence of $M$. bovis strain BCG during the extraction procedure. (SE/Ra) and (SE/ BCG), Sonic extracts of strain $\mathrm{H} 37 \mathrm{Ra}$ and BCG cells, respectively; (SE/Ra + BCG), sonic extract of a mixture of strain H37Ra and BCG cell suspensions (1:1 ratio); $(\eta)$, partially purified preparation of precipitinogen $\eta(20 \mu \mathrm{g}$ of protein per $\mathrm{ml}) ;[\mathrm{A} \eta]$, rabbit antiserum against partially purified precipitinogen $\eta$. The antigen wells contained $10 \mu \mathrm{l}$ of sonic extract ( $5 \mathrm{mg}$ of protein per $\mathrm{ml}$ ).

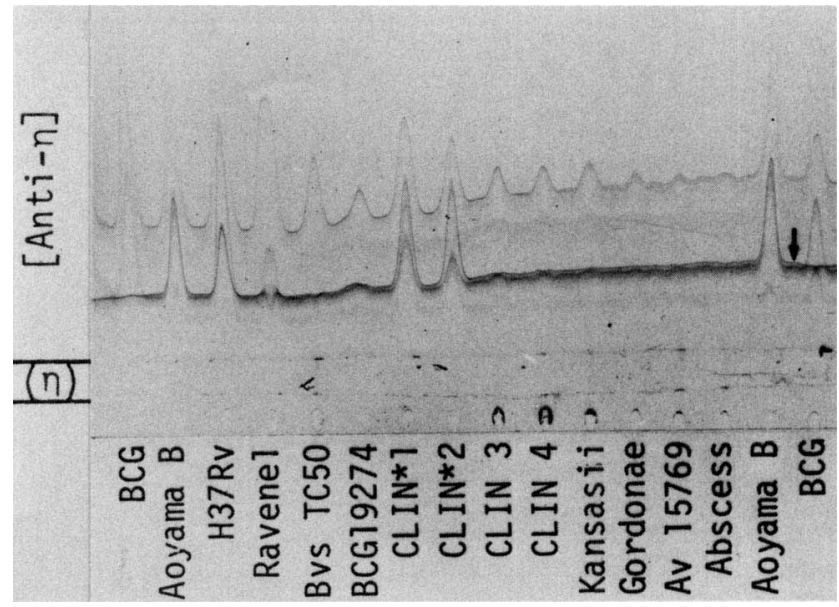

FIG. 4. Rocket line immunoelectrophoresis of sonic extracts of various species of mycobazteria. A purified preparation of precipitinogen $\eta(30 \mu \mathrm{g} / \mathrm{ml})$ was used in the intermediate gel, and its antiserum was used in the top agar. Electrophoresis was carried out overnight from bottom to top at a constant voltage of $10 \mathrm{~V} / \mathrm{cm}$ at room temperature. Portions $(3 \mu \mathrm{l})$ of sonic extracts $(2$ to $5 \mathrm{mg}$ of protein per $\mathrm{ml}$ ) of the following strains were introduced into wells: BCG, strain BCG (Biken); H37Rv, strain H37Rv (TMC 102); Bvs TC50, M. bovis TC50; BCG19274, strain BCG (ATCC 19274); CLIN 1, CLIN 2, CLIN 3, and CLIN 4, strains derived from patient sputa (asterisks indicate niacin test-positive strains); Kansasii, $M$. kansasii; Gordonae, $M$. gordonae; Av 15769, $M$. avium; Abscess, $M$. chelonei subsp. abscessus. The arrow indicates the precipitation line of $\eta$ precipitinogen.

extract, but not a $M$. bovis BCG extract, and named this precipitinogen $\eta(4,5)$. The fact that anti- $M$. bovis sera did not form a $\eta$ precipitation band indicated that $\eta$ precipitinogen was not present in $M$. bovis extracts and that the $\eta$ band formed with anti-M. tuberculosis extract serum was due to the antigen-antibody reaction. Although $\eta$ precipitinogen was clearly present in the $M$. tuberculosis H37Ra extract, our results raised the question of whether the $\eta$ band was not detectable in $M$. bovis $B C \bar{G}$. The results of this study support the idea that $\eta$ precipitinogen is in fact not present in $M$. bovis extracts, although it is still possible that the activity of the $\eta$ precipitinogen is modified or masked in $M$. bovis extracts. In any case, the results described above indicate that the system for preparing and detecting $\eta$ activity that we described previously (5) is reproducible and useful for examining the distribution of $\eta$ precipitinogen in various species of mycobacteria.

In laboratory diagnosis of mycobacteria, $M$. tuberculosis has been taxonomically differentiated from $M$. bovis by biochemical reactions, including the key niacin test, reduction of nitrate, the amidase reactions, and growth on media containing either furan-2-carboxylic acid hydrazide or thiophen-2-carboxylic acid hydrazide (2). However, no specific

TABLE 2. Distribution of $\eta$ precipitinogen in freshly isolated mycobacteria

\begin{tabular}{lccr}
\hline Origin & $\eta$ Activity & Niacin test & $\begin{array}{r}\text { No. of } \\
\text { strains }\end{array}$ \\
\hline Human & + & + & 29 \\
& - & + & 1 \\
Bovine & - & - & 13 \\
\hline
\end{tabular}


single test for the species $M$. tuberculosis is available. Strains of $M$. bovis that so far have been generally believed to be antigenically indistinguishable from strains $M$. tuberculosis are usually niacin negative, but they can become niacin positive after repeated subculture (Table 1 ), as reported by Takahashi and Murohashi (11).

The results of this study clearly show, without exception, that all mycobacterial strains other than those of $M$. tuberculosis are $\eta$ precipitinogen negative. Although Mycobacterium ulcerans frequently shows scanty growth and the cells are more resistant to sonic disintegration than the cells of other strains of mycobacteria, the protein content of sonic extracts of $M$. ulcerans examined for $\eta$ precipitinogen activity (about $1 \mathrm{mg}$ of protein per $\mathrm{ml}$ ) was always lower than the protein contents of other species $(10 \mathrm{mg}$ of protein per $\mathrm{ml})$. However, in control experiments, diluted sonic extracts of strains of $M$. tuberculosis were invariably $\eta$ precipitinogen positive, even at a protein concentration of $1 \mathrm{mg} / \mathrm{ml}$. Therefore, I concluded that $M$. ulcerans was in fact $\eta$ precipitinogen negative.

From a taxonomic point of view, it would be interesting to test for the presence of $\eta$ precipitinogen in Mycobacterium africanum, which has been reported to be a member of the tuberculous group (10). However, so far we have been unsuccessful in obtaining sufficient growth of $M$. africanum for the $\eta$ precipitinogen test by immunodiffusion. A more sensitive test for $\eta$ precipitinogen than immunodiffusion or immunoelectrophoresis may be necessary for examining this strain.

My data show that the system for detecting $\eta$ precipitinogen is applicable not only for laboratory strains but also for fresh isolates of mycobacteria, even when they might be drug resistant. With one exception, I found $\eta$ precipitinogen in all isolates that showed the niacin reaction, irrespective of drug resistance. These strains were tested within four subcultures of the original isolation (one or two subcultures in Ogawa medium and one or two subcultures onto potato blocks immersed in glycerol broth), but even repeated subcultures did not change the $\eta$-positive response, as shown with the laboratory strains (e.g., strains Kurono and BCG [Biken] that have been passaged more than 100 times). However, the procedure described above can selectively differentiate mycobacteria that exhibit a positive niacin test and can detect the presence of $\beta$ antigen purified from $M$. tuberculosis strain H37Rv culture filtrate $(14,15)$; the niacin test usually becomes positive after repeated subculturing of $M$. bovis strains (11), and $\beta$-antigen is frequently positive in INH-sensitive wild strains but negative in INH-resistant mutants of $M$. tuberculosis and $M$. bovis (15). Therefore, detection of $\eta$ precipitinogen may be useful as a single criterion for identification of $M$. tuberculosis.

Very recently, monoclonal antibodies that are apparently more specific for $M$. tuberculosis than for $M$. bovis have been reported (3). The question of whether these monoclonal antibodies are related to the antigenic determinants of $\eta$ precipitinogen remains to be investigated.

\section{ACKNOWLEDGMENTS}

I thank Morihiro Matsuda, of Tuberculosis Research Institute for Microbial Diseases, Osaka University, for fruitful discussions and help in preparing the manuscript and Hazime Saito, Department of Microbiology and Immunology, Shimane Medical College, for critical reading of the manuscript.

This work received financial support from the World Health Organization.

\section{LITERATURE CITED}

1. Barksdale, L., and K. Kim. 1977. Mycobacterium. Bacteriol. Rev, 41:217-372.

2. Bönicke, R. 1958. Die Differenzierung humaner und boviner Tuberkelbacterien mit Hilfe von Thiophen-2-carbonsäure-hydrazid. Naturwissenschaften 45:392-393.

3. Coates, A. R. M., J. Hewitt, B. W. Allen, J. Ivanyi, and D. A. Mitchinson. 1981. Antigenic diversity of Mycobacterium tuberculosis and Mycobacterium bovis detected by means of monoclonal antibodies. Lancet ii:167-169.

4. Hirai, T., Y. Shimmen, and M. Yoneda. 1979. An antigen present in strains of Mycobacterium tuberculosis. Bull. Int. Union Tuberc. 54:163.

5. Hirai, T., Y. Shimmen, and M. Yoneda. 1982. A system for preparation, detection and identification of a precipitinogen $(\eta)$ of strains of Mycobacterium tuberculosis, that is not detectable in strains of $M$. bovis. Biken J. 25:1-19.

6. Konno, K. 1956. New chemical method to differentiate humantype tubercle bacilli. Science 124:985.

7. Lind, A. 1978. Mycobacterial antigens. Ann. Microbiol. (Paris) 129 A:99-107.

8. Runyon, E. H., L. G. Wayne, and G. P. Kubica. 1974. Family II. Mycobacteriaceae Chester 1987, 63, p. 681. In R. E. Buchanan and N. E. Gibbons (ed.), Bergey's manual of determinative bacteriology, 8th ed. The Williams \& Wilkins Co., Baltimore.

9. Stanford, J. L., and A. Beck. 1968. An antigenic analysis of the mycobacteria, Mycobacterium fortuitum, Myco. kansasii, Myco. phlei, Myco. smegmatis and Myco. tuberculosis. J. Pathol. Bacteriol. 95:131-139.

10. Stanford, J. L., and J. M. Grange. 1974. The meaning and structure of species as applied to mycobacteria. Tubercle 55:143-152.

11. Takahashi, H., and T. Murohashi. 1976. Changes in biological properties by subculture of tuberculous group of mycobacteria. Kekkaku 51:227. (In Japanese.)

12. Wayne, L. G., and J. R. Doubek. 1968. Diagnostic key to mycobacteria encountered in clinical laboratories. Appl. Microbiol. 16:925-931.

13. Wilson, G. S. 1925. The serological classification of the tubercle bacilli by agglutination and adsorption of agglutinins. J. Pathol. Bacteriol. 28:69-97.

14. Yoneda, M., and Y. Fukui. 1965. Isolation, purification, and characterization of extracellular antigens of Mycobacterium tuberculosis. Am. Rev. Respir. Dis. 92:9-18.

15. Yoneda, M., Y. Fukui, and T. Yamanouchi. 1965. Extracellular proteins of tubercle bacilli. V. Distribution of $\alpha$ and $\beta$ antigens in various mycobacteria. Biken J. 8:201-223. 